\title{
Maximizing fabrication and thermal tolerances of all-silicon FIR wavelength filters
}

\author{
Sarvagya Dwivedi, Student Member IEEE, Herbert D'heer and Wim Bogaerts, Senior Member IEEE
}

\begin{abstract}
We propose a method to make silicon optical finite impulse response (FIR) filters tolerant to fabrication (waveguide geometry) and ambient thermal variations. We experimentally demonstrate a Mach-Zehnder interferometer (MZI) filter with fabrication and thermal tolerance, both separately and together. The fabrication-tolerant device measurements show a 20 -fold improved tolerance to systematic waveguide linewidth variations, with a wavelength shift of less than $60 \mathrm{pm} / \mathrm{nm}$ linewidth change. The fabrication- and thermal-tolerant device is possible by using orthogonal polarizations in the two arms. The fabricated device shows a shift of less than $\pm 65 \mathrm{pm} / \mathrm{nm}$ and a thermal drift smaller than $\pm 15 \mathrm{pm} / \mathrm{K}$ over a wavelength range of $40 \mathrm{~nm}$. Simulations show that this concept can be extended to multi-channel filters.
\end{abstract}

Index Terms - Silicon photonics, wavelength filtering devices, fabrication tolerances, athermal

\section{INTRODUCTION}

Silicon on Insulator (SOI) is a promising platform because of its high refractive index contrast that enables dense integration of micron-sized photonic integrated circuits. However, the high index contrast and submicron waveguide dimensions can translate a small change in the waveguide width and height into a large change in the refractive index [1]. This brings a weakness to this platform, i.e. extremely precise fabrication is required, especially for wavelength filtering devices. While fabrication technology improves systematically, the required fabrication control limits the yield of larger circuits.

Another important issue is the high thermo-optic coefficient of silicon $\left(1.86 \times 10^{-4} \mathrm{~K}^{-1}\right)$, which affects device performance because of ambient temperature variations.

Fabrication and thermal variations can be actively compensated (e.g. with thermal tuning), but this will drive up power consumption and complicate the device with active control circuitry. Post-fabrication trimming [2] is possible, but it significantly increases the fabrication cost.

Other techniques involve compensating materials such as polymers or $\mathrm{TiO}_{2}[3,4]$, but these again complicate fabrication and also face complementary metal-oxide-semiconductor (CMOS) compatibility and reliability issues.

We present a technique to make a wavelength filter tolerant to linewidth variations and thermal variations by design, without changing the fabrication process itself. The technique

\section{Manuscript received}

All authors are with the Photonics Research Group, Department of Information Technology, Ghent University-imec, Center of Nano-and Biophotonics, St. Pietersnieuwstraat 41, 9000 Gent, Belgium.

Wim Bogaerts is also with Luceda Photonics, 9200 Dendermonde, Belgium. E-mail:sarvagya.dwivedi@intec.ugent.be
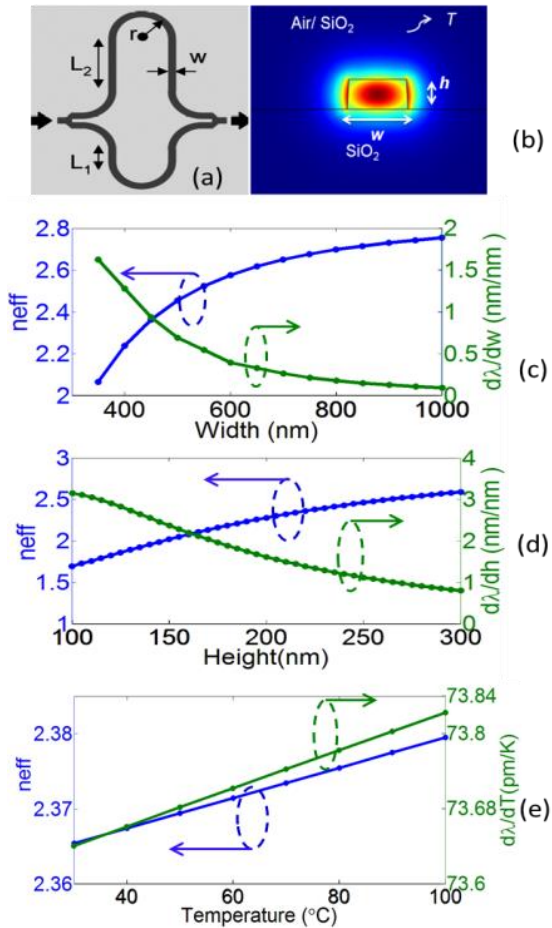

Fig. 1.(a) Schematic of an MZI filter,(b) the mode profile of a silicon waveguide of $w=450 \mathrm{~nm}, h=220 \mathrm{~nm}$ and at room temperature $T=20^{\circ} \mathrm{C}$, (c) Effective refractive index and sensitivity of spectrum position to width,(d) height and (e) temperature variation.

uses a difference in mode confinement in the two arms of the filter to passively compensate these variations [5]. We have demonstrated this technique for linewidth variations [6].

However, fabrication tolerance is not enough to make the SOI platform robust; thermal tolerance is equally important. Here we extend the technique to make filters robust against multiple effects (linewidth and thermal variations) simultaneously. Simulations show that the technique can be used for higher-order filters with multiple channels. A two-stage, four-channel filter with fabrication and thermal tolerance is simulated and discussed.

\section{PROPOSED METHOD AND DESIGN}

The wavelength sensitivity of a FIR filtering device to a parameter $X$ can be expressed as

$$
\frac{d \lambda}{d X}=\frac{\lambda_{m}}{n_{g}} \frac{d n_{e f f}}{d X}
$$

where $X$ can be the waveguide width $(w)$, height $(h)$, temperature $(T)$ or any other influence. We assume here that the influence affects the entire device uniformly. $\lambda_{m}$ is the wavelength of operation, $n_{\text {eff }}$ is the effective refractive index and $n_{g}$ is the group index. Fig. 1(a) shows the standard MZI 
filter with optical path length difference (L2-L1) and designed bend radius in arms is $r$. Fig. 1(b) shows the simulated mode profile of a standard SOI waveguide with $w=450 \mathrm{~nm}, h=220$ $\mathrm{nm}$ at a wavelength of $1550 \mathrm{~nm}$ and at $T=20^{\circ} \mathrm{C}$. The $n_{\text {eff }}$ of SOI waveguide and sensitivity of the spectral position of FIR filters made of these waveguides i.e. $(d \lambda / d w)$, height $(d \lambda / d h)$ and temperature $(d \lambda / d T)$ are shown in Figs. 1(c), (d) and (e), respectively. The wavelength shift of an MZI (plotted in green) is around $1 \mathrm{~nm}$ for a $1 \mathrm{~nm}$ change in waveguide width and is around $1.4 \mathrm{~nm}$ for $1 \mathrm{~nm}$ change of waveguide height. Fig. 1(c) also shows that the sensitivity decreases for a larger width since the waveguide converges to a slab waveguide. These variations generally true for all waveguide-based FIR filtering devices on SOI. In order to compensate the wavelength sensitivity due to the fabrication variations, we introduce design variations such as different waveguide width in each arm. The modified expression for the sensitivity then becomes

$$
\frac{d \lambda}{d X}=\frac{\lambda_{m}}{n_{g 1} L_{1}-n_{g 2} L_{2}}\left(L_{1} \frac{d n_{e f f 1}}{d X}-L_{2} \frac{d n_{e f f 2}}{d X}\right) \rightarrow 0
$$

which we try to minimize where $L_{1}, L_{2}$ are the arm lengths; $n_{g l}$, $n_{g 2}$ are the group indices, and $n_{\text {eff } 1}, n_{\text {eff } 2}$ are their corresponding effective refractive indices, respectively. For a device tolerant to width variations, the condition for zero sensitivity at wavelength $\lambda_{0}$ and for a given free spectral range (FSR) becomes

$$
\frac{L_{1}}{L_{2}}=\frac{d n_{e f f 2} / d w}{d n_{e f f 1} / d w}
$$

For the filter to be width-tolerant, the length ratio of the arms should be inversely proportional to the sensitivity of the effective index of the waveguides of the arms. Here, we assume that the linewidth change induced by the fabrication process is similar for the waveguides in the two arms. This is generally true if the linewidth change is caused by global or long-range effects, such as lithographic dose variations or a change in etch rate, and the device is sufficiently compact.

In order to make the devices fabrication- as well as thermally tolerant, we introduce orthogonal polarizations transverse electric (TE) and transverse magnetic (TM) in the two arms of the filter. The details of thermal compensation of a similar kind of device are explained in [7]. For simultaneous width- and thermal-tolerant, the wavelength sensitivity with respect to temperature ( $d \lambda / d T)$ becomes zero and (2) should satisfy

$$
L_{T M} \frac{d n_{e f f, T M}}{d T}=L_{T E} \frac{d n_{e f f, T E}}{d T}
$$

where $L_{T M}$ and $L_{T E}$ are lengths of the TM and TE arms and $d n_{e f f, T M} / d T$ and $d n_{e f f, T E} / d T$ their corresponding waveguide thermo-optic (TO) coefficients. The combined condition of being tolerant to width and temperature at wavelength $\lambda_{0}$ and at temperature $T_{0}$ for a given FSR becomes

$$
\frac{L_{T E}}{L_{T M}}=\frac{T O_{T M}}{T O_{T E}}=\frac{d n_{e f f, T M} / d w}{d n_{e f f, T E} / d w}
$$

Physically it means that a variation in width or temperature gives an identical change in phase in each arm. The simulated contour plot of the squared sensitivity of the spectral position to the width for an athermal MZI is shown in Fig.2 with respect to the TE and TM core widths. The simulation shows that there is

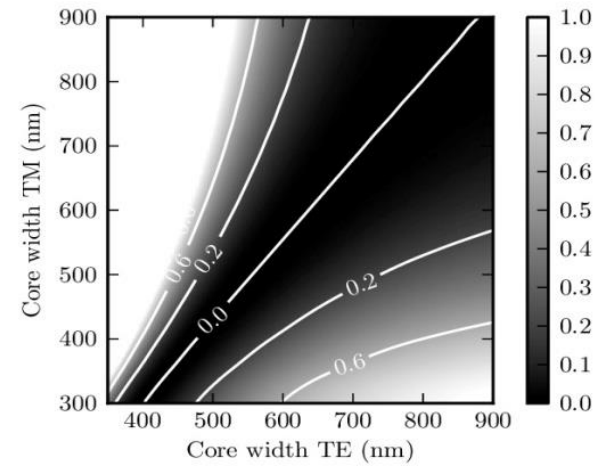

Fig. 2. $(d \lambda / d w)^{2}$ of the athermal MZI filter at $\lambda=1550 \mathrm{~nm}$ and $\mathrm{T}=20^{\circ} \mathrm{C}$.

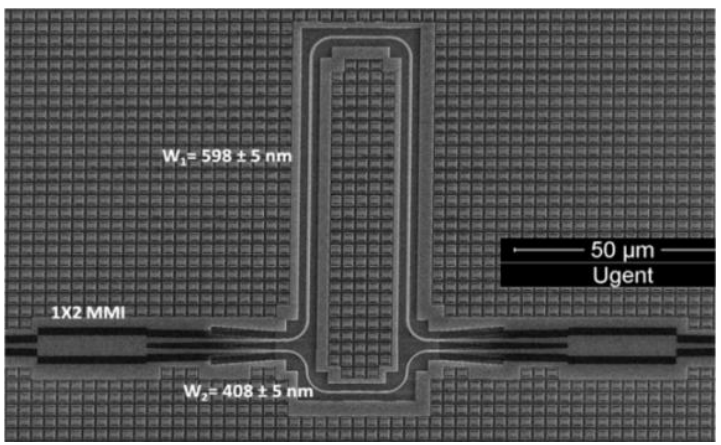

Fig. 3. SEM image of fabricated MZI filter with shallow-etch MMIs.

a region where the sensitivity due to linewidth variations of waveguide is minimized. Although the actual value of sensitivity could be negative, the squared sensitivity contour plot gives a better picture of where the minimal region is located. This method can be further generalized to width, height and temperature. A filter that is tolerant to all the three parameters could be constructed by using arms with different polarizations and consisting of multiple sections with different widths. When a component has to be athermal and tolerant to width and height at the same time, then the condition $\frac{d \lambda_{m}}{d T}=$ $\frac{d \lambda_{m}}{d w}=\frac{d \lambda_{m}}{d h}=0$, has to be fulfilled in the temperature and the wavelength region of interest. We focus in this paper on the compensation of linewidth variations (primarily caused by etching and lithography) and ambient thermal fluctuations.

\section{FABRicATION AND MEASUREMENTS}

We have designed the devices using the IPKISS parametric design framework [9]. The fabricated MZI filter scanning electron microscope (SEM) image is shown in Fig.3. The filter uses a shallow-etch multimode interference (MMI) coupler as a $50 / 501 \times 2$ power splitter, and the same is used as a combiner for a broadband operation [8]. In order to demonstrate the width-tolerant behavior given in equation (3) the designed widths of upper and lower arms are $600 \mathrm{~nm}$ and $400 \mathrm{~nm}$ and their corresponding designed lengths are $172 \mu \mathrm{m}$ and $45 \mu \mathrm{m}$, respectively. The devices are fabricated in IMEC's standard passive platform using the ePIXfab MPW service. This process uses $200 \mathrm{~mm}$ SOI wafers with nominally $220 \mathrm{~nm}$ of silicon and $2 \mu \mathrm{m}$ of buried oxide. In order to demonstrate the tolerant behavior we replicate the device with deliberate relative variations in the linewidth in both arms over a $20 \mathrm{~nm}$ range. A 


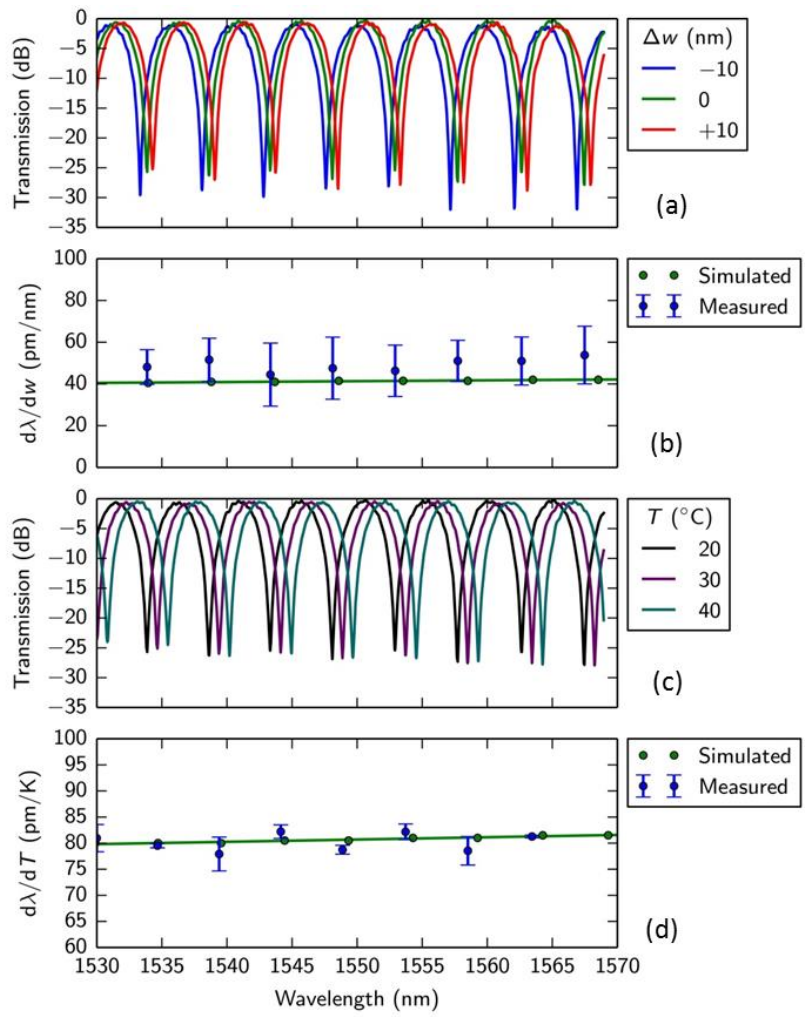

Fig. 4. (a) Measured transmission of the optimized design for $0,-10$ and +10 nm width offset $\mathrm{T}=20^{\circ} \mathrm{C}$, (b) width sensitivity $(\mathrm{d} \lambda / \mathrm{d} w)$ variation simulated and measured, (c) measured transmission at $\mathrm{T}=20,30$ and $40{ }^{\circ} \mathrm{C}$ (d) thermal sensitivity $(\mathrm{d} \lambda / \mathrm{d} T)$ variation simulated and measured.

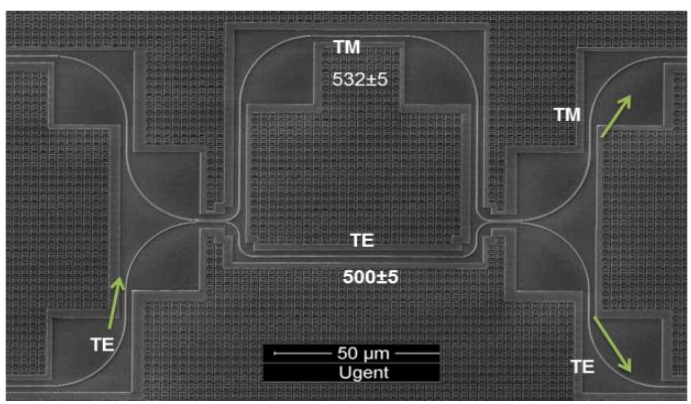

Fig. 5. SEM image of fabricated MZI filter with SPRs

$\pm 10 \mathrm{~nm}$ absolute linewidth variation can be considered as controllable for an optimized, stabilized fabrication process.

The measured thickness of the silicon waveguide in the presented devices is $215 \mathrm{~nm}$. The measured arm width (from SEM inspection) is approximately $598 \pm 5 \mathrm{~nm}$ in the upper arm and $408 \pm 5 \mathrm{~nm}$ in the lower arm, as shown in Fig.3. The measured transmission spectra of this device for width offset of $0,-10$ and $+10 \mathrm{~nm}$ and temperature controlled environment at $20{ }^{\circ} \mathrm{C}$ are shown in Fig. 4(a). The devices show an extinction ratio of more than $25 \mathrm{~dB}$ and an insertion loss of less than 0.3 $\mathrm{dB}$ throughout the $\mathrm{C}$ band.

The measured FSR of the filter is around $5 \mathrm{~nm}$. The extracted and simulated sensitivity to linewidth $d \lambda / d w$ is shown in Fig. 4(b). Device simulations are performed using circuit solver Caphe [10] and the results match very well with measurements. The measured width sensitivity is reduced from $\sim 1 \mathrm{~nm} / \mathrm{nm}$ to less than $60 \mathrm{pm} / \mathrm{nm}$ over the entire spectrum with a standard deviation of about $15 \mathrm{pm} / \mathrm{nm}$ which is measured over four
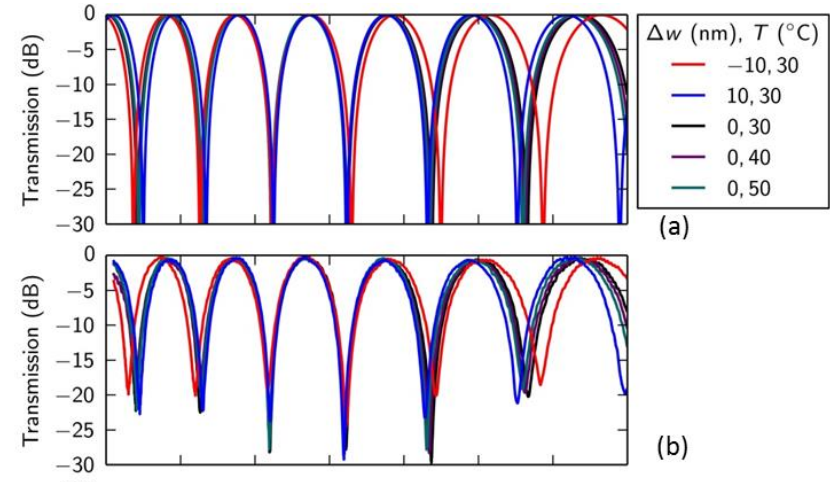

(a)
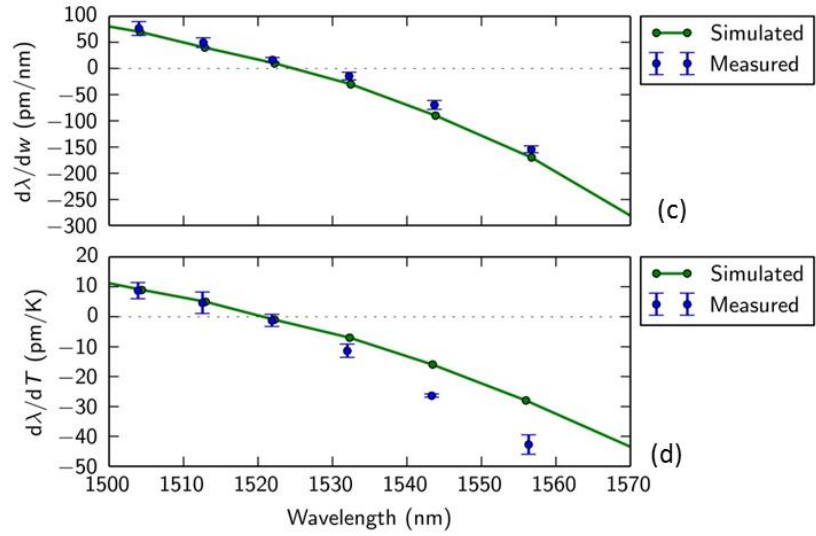

Fig. 6. (a) Simulated spectra of optimized design for $0,-10$ and $+10 \mathrm{~nm}$ width offset and at 30,40 and $50{ }^{\circ} \mathrm{C}$, (b) measured transmission, (c) width sensitivity $(\mathrm{d} \lambda / \mathrm{d} w)$ and $(\mathrm{d})$ thermal sensitivity $(\mathrm{d} \lambda / \mathrm{dT})$ simulated and measured .

devices with deliberate width offsets of $\pm 5 \mathrm{~nm}$ and $\pm 10 \mathrm{~nm}$. These measured devices are positioned close together on the chip to minimize non-intentional relative linewidth variations, which can be assumed to be of the order of $1 \mathrm{~nm}$ [1]. Measured spectra of the devices at varying temperature are shown in Fig. 4(c) and the corresponding thermal sensitivity values over the entire spectrum are shown in Fig. 4(d), respectively. Thermal measurement of the device shows that it behaves similarly to a standard MZI, with $80 \mathrm{pm} / \mathrm{K}$ of shift.

In order to demonstrate both linewidth and thermal tolerance in the same device, we should minimize the temperature dependence for devices on the minimum curve of the linewidth sensitivity (i.e. the zero diagonal in Fig. 2). We find this for a $\mathrm{TM}$ and TE arm nominal width of $490 \mathrm{~nm}$ and $522 \mathrm{~nm}$, and corresponding lengths of $180 \mu \mathrm{m}$ and $87 \mu \mathrm{m}$, which gives an FSR of $10 \mathrm{~nm}$. The SEM picture of the fabricated device is shown in Fig. 5. The device uses a splitter polarization rotator (SPR) that splits the light into two equal parts and at the same time rotates the polarization in one arm [7]. This directional coupler device is based on the phase-matching condition of a narrow TE waveguide and a wide TM waveguide $\left(n_{\text {effTE }}=\right.$ $n_{\text {effTM }}$ ). The same SPR design is used at the output to combine the two modes. The measured SEM width of the fabricated device is $502 \pm 5 \mathrm{~nm}$ in the TM arm and $534 \pm 5 \mathrm{~nm}$ in the TE arm. The simulated spectra of transmission using the device parameters from SEM are shown in Fig. 6(a). The measurement is performed at three different temperatures, i.e. 30, 40 and 50 ${ }^{\circ} \mathrm{C}$ on a nominal-width device. The transmission measurement is performed on varying width devices of $\pm 10 \mathrm{~nm}$ at $30^{\circ} \mathrm{C}$. The five measured thermal transmission spectra are shown in Fig. 


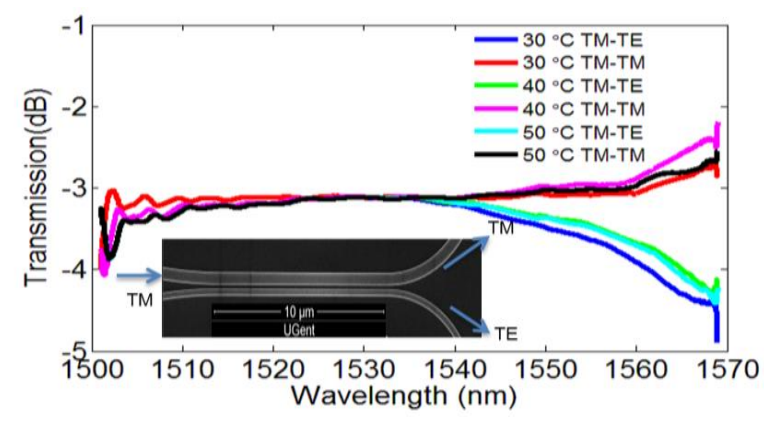

Fig.7. Bar and cross port measured transmission of SPR at different temperatures. SEM image of SPR (inset).

6(b). The fabrication and insensitive point is situated around $1525 \mathrm{~nm}$. The measured device shows a width sensitivity of less than $\pm 65 \mathrm{pm} / \mathrm{nm}$, with a standard deviation of about $15 \mathrm{pm} / \mathrm{nm}$ measured over four devices with width offsets of $\pm 10 \mathrm{~nm}$ and $\pm 20 \mathrm{~nm}$, and a thermal sensitivity of less than $\pm 15 \mathrm{pm} / \mathrm{K}$, with a standard deviation of about $3 \mathrm{pm} / \mathrm{K}$ over the wide wavelength range of $40 \mathrm{~nm}$ from 1501 to $1540 \mathrm{~nm}$. The simulated and measured width as well as the thermal sensitivity are shown in Figs. 6(c) and (d). The width sensitivity changes from positive to negative on the different sides of the insensitivity point. A similar behavior can be seen while varying the temperature of the device. This phenomenon is due to the $n_{\text {eff }}$ variation with $w$ $\left(d n_{\text {eff }} / d w\right)$ and $T\left(d n_{\text {eff }} d d T\right)$ for the TM waveguide with varying wavelength. This variation changes at a much faster rate than in the TE waveguide. If we compare the response with that of the width-tolerant device as shown in Fig. 4, the wider waveguide is always red-shifted over the entire wavelength range and the width sensitivity is much flatter. The measured transmission of the SPR at different temperatures is shown in Fig. 7. The SPR is designed for $50 \%$ power splitting in the $\mathrm{C}$ band, but we do see a deviation from that value for longer wavelengths. This will not significantly affect the athermal/width-tolerant behavior, but it will result in a lower extinction ratio of the MZI.

\section{HIGHER-ORDER FILTERING APPLICATIONS}

A schematic of a four-channel de-multiplexer is shown in Fig. 8 (a). The design is based on the width- and thermal -tolerant MZI filter. The designed channel spacing of the de-multiplexer is $6.4 \mathrm{~nm}$. The lengths of the TE and TM arms for the first stage of the device are $74.4 \mu \mathrm{m}$ and $162.9 \mu \mathrm{m}$, respectively, which are designed for the FSR of $12.8 \mathrm{~nm}$. The lengths of the arms become half in the second stage since the FSR of each MZI is doubled. The coupling coefficient $k$ of directional coupler is 0.5 , which is similar to that of the SPR, as explained in the previous section. The simulated circuit transmission at three different widths of $(0,-10,10) \mathrm{nm}$ and at temperatures of 30 and $50{ }^{\circ} \mathrm{C}$ are shown in Fig. 8(b). The simulated width and thermal sensitivity for this filter are found to be $30 \mathrm{pm} / \mathrm{nm}$ and $8 \mathrm{pm} / \mathrm{K}$, respectively. Another approach to decrease the thermal sensitivity is to flatten the transmission pass-bands as demonstrated in [11].

\section{CONCLUSION}

We have proposed and demonstrated a technique to make all-silicon tolerant FIR filter devices. The measured results show a reduced linewidth sensitivity of less than $60 \mathrm{pm} / \mathrm{nm}$ over a wide band, which is a 20 -fold improvement compared to

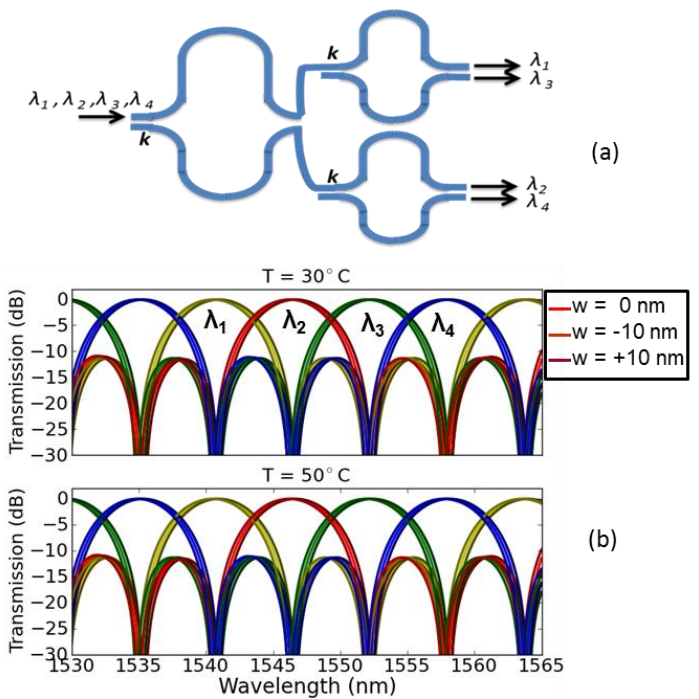

Fig. 8.(a) Schematic of two-stage filter as a 4-channel de-multiplexer with $k=$ 0.5 , (b) simulated transmission of filter at $\mathrm{T}=30^{\circ} \mathrm{C}$ and $50{ }^{\circ} \mathrm{C}$ and with width variation of $(-10,0,+10) \mathrm{nm}$.

conventional devices. We have also demonstrated a width- and thermal-tolerant compact device with a width sensitivity less than $\pm 65 \mathrm{pm} / \mathrm{nm}$ and a thermal sensitivity of smaller than \pm 15 $\mathrm{pm} / \mathrm{K}$ over a $40 \mathrm{~nm}$ of wavelength range. These devices can be used for higher-order wavelength-division-multiplexing (WDM) filters, as shown in the simulations of a four-channel thermal- and fabrication-tolerant de-multiplexer, and hence open a new set of devices where the improvement is done just at the design level itself without altering the fabrication process.

\section{ACKNOWLEDGMENT}

The authors acknowledge Liesbet Van Landschoot for SEM. This work is supported by BOF grant of Ghent University.

\section{REFERENCES}

[1] S. Selvaraja, et al., "Sub-nanometer linewidth uniformity in silicon nano-photonic waveguide devices using CMOS fabrication technology," IEEE J. Sel. Top. Quantum Electron, vol. 16, pp.316 - 324, 2010

[2] J. Schrauwen, et al., "Trimming of silicon ring resonator by electron beam induced compaction and strain," Opt. Exp., vol. 16, pp.3738-3743, 2008

[3] J. Teng, et al, "Athermal Silicon-on-insulator ring resonators by overlaying a polymer cladding on narrowed waveguides," Opt. Exp., vol. 17, pp. 14627-14633, 2009

[4] B. Guha, et al., "Athermal silicon microring resonators with titanium oxide cladding," Opt. Exp. , vol. 21, pp. 26557-26563, 2013

[5] M. Uenuma et al., "Temperature-independent silicon waveguide optical filter," Opt. Lett., vol. 34, pp. 599-601 , 2009

[6] S. Dwivedi, et. al, "Fabrication tolerant MZI filter," in Group IV Photonics 2014, pp.147-148, 2014

[7] S. Dwivedi, et. al, "A compact all-silicon temperature insensitive filter for WDM and bio-sensing applications," IEEE Photonic. Tech. Lett. , vol. 25, pp. $2167-2170,2013$

[8] W. Bogaerts, et al., "Silicon-on-insulator spectral filters fabricated with CMOS technology, " IEEE J. Sel. Topics Quantum Electron., vol. 16, no. 1 , pp. 33-44, 2010.

[9] W. Bogaerts, et al., "Integrated design for integrated photonics: From the physical to the circuit level and back," Proc. SPIE, vol. 8781,pp. 878102-1-878102-11, 2013

[10] M. Fiers, et. al., "Time-domain and frequency-domain modeling of nonlinear optical components on circuit-level using a node-based approach," J. Opt. Soc. Am. B, vol. 29, pp.896-900, 2012

[11] F. Horst, et al., "Cascaded Mach-Zehnder wavelength fiters in silicon photonics for low loss and at pass-band WDM (de-)multiplexing," Opt. Exp., vol. 21, no. 10, pp. 11652, 2013 